\title{
Association between gestational weight gain and exclusive breast-feeding for the first 6 months postpartum in Chinese women
}

\author{
Mingkun Tong ${ }^{1,2}$, Lei Jin 1,2,*, Jianmeng Liu ${ }^{1,2}$, Zhiwen $\mathrm{Li}^{1,2}$, Hongtian $\mathrm{Li}^{1,2}$, Le Zhang ${ }^{1,2}$, \\ Rongwei $\mathrm{Ye}^{1,2}$ and Aiguo Ren ${ }^{1,2}$ \\ 'Department of Epidemiology and Biostatistics, School of Public Health, Peking University, Beijing 100191, People's \\ Republic of China: ${ }^{2}$ Institute of Reproductive and Child Health, Peking University/National Health Commission of the \\ People's Republic of China Key Laboratory of Reproductive Health, Peking University, Beijing 100191, People's \\ Republic of China
}

Submitted 12 September 2018: Final revision received 10 January 2019: Accepted 18 January 2019: First published online 12 March 2019

\begin{abstract}
Objective: The association between gestational weight gain (GWG) and exclusive breast-feeding (EBF) practices remains unclear. The present study evaluated the association between GWG and EBF in the first 6 months postpartum among primiparas in rural China.

Design: The study population was drawn from a previous randomized controlled trial, and the relevant data were obtained from an electronic, population-based perinatal system and a monitoring system for child health care. GWG was categorized according to the guidelines of the Institute of Medicine.

Setting: Five rural counties in Hebei Province, China.

Participants: A total of 8449 primiparas.

Results: Of the women, $58.7 \%$ breast-fed exclusively for the first 6 months postpartum. Overweight women who gained either more or less weight than the recommended GWG tended to experience failure of $\mathrm{EBF}(\mathrm{OR}=0.49 ; 95 \% \mathrm{CI} 0.34$, $0.70 ; P<0.001$ and $\mathrm{OR}=0.79 ; 95 \%$ CI 0.63, 0.99; $P=0.048$, respectively). The same results were also observed among obese women; the OR for lower and greater weight gain were 0.28 (95\% CI 0.08, 0.94; $P=0.04)$ and 0.55 (95\% CI 0.32, $0 \cdot 95 ; P=0 \cdot 03$ ), respectively.

Conclusions: GWG that is below or above the Institute of Medicine recommendations is associated with EBF behaviour for the first 6 months postpartum in overweight and obese primiparas in rural China.
\end{abstract}

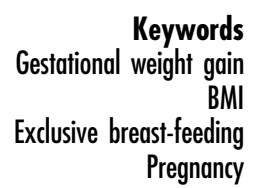

Pregnancy
The WHO recommends exclusive breast-feeding (EBF) for the first 6 months of life, followed by continued breastfeeding with appropriate complementary foods for 2 years or more ${ }^{(1)}$. The benefits of breast-feeding for both mother and child have been widely recognized, serving as a nutritional and energy supplement, helping to prevent infections and diseases, and promoting maternal wellbeing ${ }^{(2)}$. Breast-feeding helps reduce the risk for breast and ovarian carcinoma and type 2 diabetes in mothers ${ }^{(3)}$, and EBF during the first 6 months of life reduces the risk of becoming overweight in the offspring ${ }^{(4)}$ and can prevent such infections as rotavirus diarrhoea and hand, foot and mouth disease ${ }^{(5,6)}$. Globally, $37 \%$ of infants younger than 6 months in low-income and middle-income countries receive $\mathrm{EBF}^{(7)}$. However, in China, the weighted rate of EBF under 6 months was found to be only $18.6 \%$ in 2013 according to a representative national survey ${ }^{(8)}$. Moreover, there are large differences in the rates of EBF among geographic areas and ethnicities, from $2 \cdot 6$ to $72 \cdot 1 \%{ }^{(9-14)}$.

Several factors may influence successful breast-feeding practices, including factors related to the mother such as maternal age, level of education, ethnicity, smoking status, mode of delivery and work status postpartum, as well as childbirth factors such as prematurity and birth weight $^{(15-19)}$. Another factor that may be associated with suboptimal breast-feeding practices is gestational weight gain (GWG). For example, among women who are overweight or obese before pregnancy, those with GWG greater or less than the recommended amount are at higher risk of an inability to sustain EBF or full breastfeeding (and might provide the infant with water, waterbased drinks or fruit juice) ${ }^{(20-22)}$. In one study, women with GWG that was greater or less than recommendations breast-fed (providing any breast milk) nearly 1 week less 
than those with optimal weight gain ${ }^{(23)}$. However, studies have also indicated that GWG is not significantly associated with the duration of full or any breast-feeding after adjusting for confounding variables ${ }^{(24-26)}$ or when prepregnancy $\mathrm{BMI}$ is included in models ${ }^{(27)}$. Few studies have focused on the Chinese population: in one such study of a single birth cohort in urban cities, no differences were found in the duration of EBF according to $\mathrm{GWG}^{(28)}$. Thus, the association between GWG and EBF practices in China remains unclear.

The prevalence of overweight and obesity has increased dramatically among reproductive-age Chinese women and mean GWG has also increased, from $10 \cdot 1 \mathrm{~kg}$ in 1993 to $14.0 \mathrm{~kg}$ in $2010^{(29)}$. As a pregnancy factor that is more easily managed, GWG plays an essential role in maternal and child health care. To evaluate the association between GWG and EBF practices among Chinese women, data from the study population of a previous randomized controlled trial were used; the data were taken from two electronic information systems containing information on the population.

\section{Methods}

The randomized controlled trial enrolled 18775 pregnant women with no or mild anaemia from five rural counties of Hebei Province, northern China, from May 2006 through April 2009 and investigated the effects of micronutrient supplements on maternal and infant health. The inclusion criteria required that the women were nulliparous, $\geq 20$ years old, had recorded dates of menstruation for $\geq 2$ months before pregnancy, had not reached 20 weeks' gestation at the time of enrolment, had not consumed micronutrient supplements other than folic acid in the previous 6 months, had an Hb level greater than 10.0 g/dl, resided in and received prenatal care in one of the five target counties (Yuanshi, Mancheng, Xianghe, Fengrun and Laoting), were legally competent and consented to participate $^{(30)}$. The study population for the current study was drawn from this trial. Data from two electronic surveillance systems for the study population were extracted for analysis. One was a routine electronic perinatal system that was established to monitor pregnant women from their first prenatal care visit to $42 \mathrm{~d}$ after delivery. The demographic characteristics, prenatal examination records and delivery summaries were obtained, including information on maternal age, height, education, ethnicity, occupation, age at menarche, gravidity, mode of delivery, gestational week at delivery, infant sex and infant birth weight. The gestational week and current weight were registered in the system using standard devices and procedures at every prenatal care visit. The other was a monitoring system for child health care with data from birth to the child's first birthday. Doctors collected data on feeding patterns when the mothers took their children to clinics for examinations and vaccinations, and the information obtained was concentrated mainly around months 3, 6, 9 and 12 postpartum.

A flow diagram of population selection is given in Fig. 1. Birth outcomes were known for 17897 of the pregnant women (twenty-eight moved away permanently, 815 had spontaneous or induced abortions, thirty-three dropped

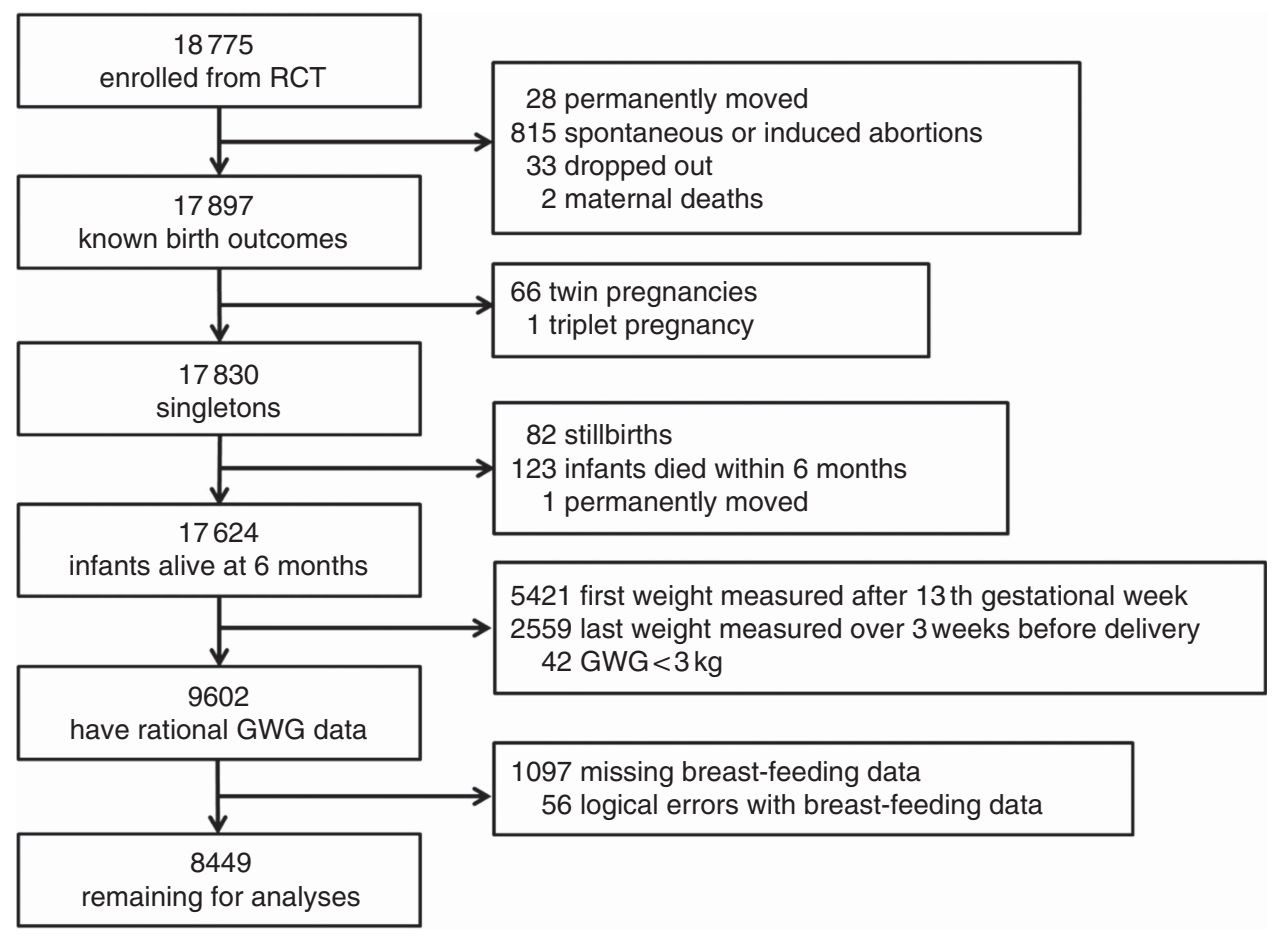

Fig. 1 Flow diagram showing the selection of the study population (RCT, randomized controlled trial; GWG, gestational weight gain) 
out of the study and two mothers died). We excluded records with multiple births ( $n$ 67), stillbirths ( $n$ 82), infants who died within 6 months or with mothers who had permanently moved ( $n$ 124). Some crucial information was missing, including data for women whose first weight was measured after the first trimester ( $>13$ weeks' gestation, $n$ 5421) and whose last weight was measured over 3 weeks before delivery ( $n$ 2559), as well as data on the breast-feeding situation during the first 6 months ( $n$ 1097). In addition, we excluded forty-two records with biologically implausible values for GWG $(<3 \mathrm{~kg})$ and fifty-six inaccurate records, such as women who reported EBF at the 6th month but had already reported having discontinued breast-feeding. Finally, 8449 pregnant women were included for analysis. We compared the main demographic characteristics between the women who were included in the study and those who were excluded. The results are shown in the online supplementary material (Supplemental Table 1). Although the differences between characteristics, with the exceptions of maternal age and infant sex, were statistically significant, they were nevertheless small and presumably biologically unimportant.

The outcome variable for the present study was EBF for the first 6 months postpartum, defined as no liquids besides human milk and no solid food given to the infant. Due to variable times of doctor visits postpartum, the breast-feeding patterns that were reported from months 5 to 7 were used. That is, if the breast-feeding patterns at the 6th month were not available, reports at month 7 were used, if available. Otherwise, the breast-feeding patterns for month 5 were used.

The variable of primary interest was GWG, which was calculated as the difference between weight at the last prenatal visit measured 3 weeks before delivery or later and weight at the first prenatal visit during the first trimester. The 2009 Institute of Medicine recommendations guided the categorization of GWG: low GWG $(<12 \cdot 5$, $<11 \cdot 5,<7 \cdot 0$ and $<5.0 \mathrm{~kg}$ for underweight, normal-weight, overweight and obese women, respectively), high GWG ( $>18 \cdot 0,>16 \cdot 0,>11.5$ and $>9 \cdot 0 \mathrm{~kg}$ for underweight, normalweight, overweight and obese women, respectively) and optimal GWG (all weight gains between the cut-offs for low and high GWG). Pre-pregnancy BMI was calculated from the weight and height at the first prenatal visit measured at enrolment in the trial; BMI was categorized as underweight $\left(<18.5 \mathrm{~kg} / \mathrm{m}^{2}\right)$, normal weight $\left(18.5-23.9 \mathrm{~kg} / \mathrm{m}^{2}\right)$, overweight $\left(24.0-27.9 \mathrm{~kg} / \mathrm{m}^{2}\right)$ or obese $\left(\geq 28.0 \mathrm{~kg} / \mathrm{m}^{2}\right)$, following the Chinese criteria.

Other demographic characteristics were transformed into categorical variables, such as maternal age (20-22, 23-25 and $\geq 26$ years old), educational level (high school or above, middle school, and primary school or less), occupation (farmer/others), ethnicity (Han/others), age at menarche ( $\leq 13,14$ and $\geq 15$ years), gravidity ( 1 and $\geq 2$ ), caesarean (yes/no), preterm (yes/no), infant sex (male/female) and infant birth weight (<2500, 2500-4000 and $\geq 4000 \mathrm{~g})$.

\section{Statistical analysis}

Descriptive statistics were generated for the demographic factors. To compare data for the women who breast-fed exclusively for the first 6 months and those who did not, $\chi^{2}$ tests were used. Two multivariable logistic regressions were performed to assess whether GWG was associated with EBF. The first calculated the OR for successful EBF for the first 6 months when only the GWG category was considered, and selected optimal weight gain as a reference. Given that the recommendations for appropriate weight gain during pregnancy are created according to pre-pregnancy BMI in clinical practice, a second logistic regression was also used to examine the OR for each category of pre-pregnancy BMI with the optimal range serving the reference group. Variables with significant statistical differences in $\chi^{2}$ tests were adjusted for education level, gravidity, caesarean and preterm in both regression analyses. OR with $95 \% \mathrm{CI}$ are given in the results. All $P$ values reported were twotailed, and a significance level of $5 \%$ was adopted $(P<0.05)$. All statistical analyses were conducted with the statistical software package IBM SPSS Statistics version 20.0 .

\section{Results}

Half of the study population was between 20 and 22 years old, the majority were farmers, of Han ethnicity and had a middle-school education. Almost two-thirds of the women (73.6 \%) had normal pre-pregnancy BMI, 20.1\% were beyond the appropriate range $(16.5 \%$ overweight and $3.6 \%$ obese) and $6.3 \%$ were below it. Less than half ( $40.4 \%)$ gained weight within the recommended amount during pregnancy, with $36.0 \%$ gaining less and $23.6 \%$ gaining more than the Institute of Medicine recommendation. At month $6,83.4 \%$ of women reported breastfeeding, and the EBF rate for 6 months postpartum was $58.7 \%$. Mothers with higher levels of education, who had first-time pregnancies, and who had a vaginal delivery and preterm labour had successful EBF for the first 6 months (Table 1).

In the first logistic regression model (Table 2), women who gained excessive weight during pregnancy had no association with successful EBF relative to women with optimal weight gain, but those who gained less weight than recommended had significantly lower odds of successful EBF for 6 months (OR=0.85; $95 \%$ CI 0.77, 0.94; $P=0.002)$. The more detailed classification shown by the second logistic regression model (Table 2) indicated that overweight women who gained either more or less weight than the recommended amount failed to perform EBF on the level of those with optimal GWG (OR $=0.49$; $95 \%$ CI $0.34,0.70 ; P<0.001$ and $\mathrm{OR}=0.79 ; 95 \% \mathrm{CI} 0.63,0.99$; $P=0.048$, respectively). The same results were also observed among obese women, with OR of less and 
Table 1 Characteristics of the pregnant women included in the study categorized by successful exclusive breast-feeding for the first 6 months ( $n$ 8449); primiparas from five rural counties in Hebei Province, China, May 2006April 2009

\begin{tabular}{|c|c|c|c|c|c|c|c|}
\hline \multirow[b]{3}{*}{ Characteristic } & & & \multicolumn{4}{|c|}{ Successful EBF } & \multirow[b]{3}{*}{$P$ value } \\
\hline & \multicolumn{2}{|c|}{ Total $^{*}$} & \multicolumn{2}{|c|}{ Yes (n 4962) } & \multicolumn{2}{|c|}{ No $(n 3487)$} & \\
\hline & $n$ & $\%$ & $n$ & $\%$ & $n$ & $\%$ & \\
\hline \multicolumn{8}{|l|}{ Maternal age (years) } \\
\hline 20-22 & 4266 & $50 \cdot 5$ & 2522 & $50 \cdot 8$ & 1744 & $50 \cdot 0$ & \multirow[t]{3}{*}{0.34} \\
\hline $23-25$ & 2849 & 33.7 & 1643 & $33 \cdot 1$ & 1206 & 34.6 & \\
\hline$\geq 26$ & 1334 & $15 \cdot 8$ & 797 & $16 \cdot 1$ & 537 & $15 \cdot 4$ & \\
\hline \multicolumn{8}{|l|}{ Education } \\
\hline Primary school or less & 116 & 1.4 & 55 & $1 \cdot 1$ & 61 & 1.8 & \multirow[t]{3}{*}{0.006} \\
\hline Middle school & 6872 & 81.3 & 4013 & 80.9 & 2859 & $82 \cdot 0$ & \\
\hline High school or above & 1461 & $17 \cdot 3$ & 894 & $18 \cdot 0$ & 567 & $16 \cdot 3$ & \\
\hline \multicolumn{8}{|l|}{ Occupation } \\
\hline Farmer & 7817 & 92.5 & 4611 & 92.9 & 3206 & 91.9 & \multirow[t]{2}{*}{0.09} \\
\hline Other & 632 & 7.5 & 351 & $7 \cdot 1$ & 281 & $8 \cdot 1$ & \\
\hline \multicolumn{8}{|l|}{ Ethnicity } \\
\hline Han & 8369 & $99 \cdot 0$ & 4907 & $98 \cdot 9$ & 3462 & $99 \cdot 3$ & \multirow[t]{2}{*}{0.067} \\
\hline Other & 80 & 1.0 & 55 & 1.1 & 25 & 0.7 & \\
\hline \multicolumn{8}{|c|}{ Age at menarche (years) } \\
\hline$\leq 13$ & 2303 & $27 \cdot 3$ & 1332 & $26 \cdot 9$ & 971 & $27 \cdot 8$ & \multirow[t]{3}{*}{0.142} \\
\hline 14 & 3232 & 38.3 & 1876 & 37.8 & 1356 & 38.9 & \\
\hline$\geq 15$ & 2913 & 34.5 & 1753 & $35 \cdot 3$ & 1160 & $33 \cdot 3$ & \\
\hline \multicolumn{8}{|l|}{ Gravidity } \\
\hline 1 & 8089 & $95 \cdot 7$ & 4804 & $96 \cdot 8$ & 3285 & $94 \cdot 2$ & \multirow[t]{2}{*}{$<0.001$} \\
\hline$\geq 2$ & 360 & 4.3 & 158 & 3.2 & 202 & $5 \cdot 8$ & \\
\hline \multicolumn{8}{|l|}{ Caesarean } \\
\hline No & 4116 & $48 \cdot 8$ & 2712 & $54 \cdot 8$ & 1404 & $40 \cdot 3$ & \multirow[t]{2}{*}{$<0.001$} \\
\hline Yes & 4311 & $51 \cdot 2$ & 2232 & $45 \cdot 2$ & 2079 & 59.7 & \\
\hline \multicolumn{8}{|l|}{ Preterm } \\
\hline No & 8041 & $95 \cdot 2$ & 4703 & $94 \cdot 8$ & 3338 & $95 \cdot 7$ & \multirow[t]{2}{*}{0.046} \\
\hline Yes & 408 & $4 \cdot 8$ & 259 & $5 \cdot 2$ & 149 & $4 \cdot 3$ & \\
\hline Infant sex & & & & & & & \\
\hline Male & 4411 & $52 \cdot 2$ & 2592 & $52 \cdot 2$ & 1819 & $52 \cdot 2$ & 0.948 \\
\hline Female & 4038 & $47 \cdot 8$ & 2370 & $47 \cdot 8$ & 1668 & 47.8 & \\
\hline Birth weight (g) & & & & & & & \\
\hline$<2500$ & 142 & 1.7 & 74 & 1.5 & 68 & $2 \cdot 0$ & 0.263 \\
\hline $2500-4000$ & 7959 & 94.2 & 4681 & 94.3 & 3278 & 94.0 & \\
\hline$\geq 4000$ & 348 & $4 \cdot 1$ & 207 & 4.2 & 141 & 4.0 & \\
\hline Pre-pregnancy BMI† & & & & & & & \\
\hline Underweight & 531 & $6 \cdot 3$ & 316 & $6 \cdot 4$ & 215 & $6 \cdot 2$ & 0.683 \\
\hline Normal & 6220 & $73 \cdot 6$ & 3670 & 74.0 & 2550 & $73 \cdot 1$ & \\
\hline Overweight & 1395 & $16 \cdot 5$ & 804 & $16 \cdot 2$ & 591 & $17 \cdot 0$ & \\
\hline Obese & 303 & 3.6 & 172 & 3.5 & 131 & 3.8 & \\
\hline GWG‡ & & & & & & & \\
\hline Less & 3044 & $36 \cdot 0$ & 1727 & $34 \cdot 8$ & 1317 & $37 \cdot 8$ & 0.014 \\
\hline Optimal & 3410 & 40.4 & 2056 & 41.4 & 1354 & $38 \cdot 8$ & \\
\hline Excessive & 1995 & $23 \cdot 6$ & 1179 & $23 \cdot 8$ & 816 & $23 \cdot 4$ & \\
\hline
\end{tabular}

GWG, gestational weight gain.

*Total may vary according to the presence of missing values in some characteristics. †BMI was categorized as underweight $\left(<18.5 \mathrm{~kg} / \mathrm{m}^{2}\right)$, normal weight $\left(18.5-23.9 \mathrm{~kg} / \mathrm{m}^{2}\right)$, overweight $\left(24.0-27.9 \mathrm{~kg} / \mathrm{m}^{2}\right)$ or obese $\left(\geq 28.0 \mathrm{~kg} / \mathrm{m}^{2}\right)$.

fLow GWG, $<12.5,<11.5,<7.0$ and $<5.0 \mathrm{~kg}$ for underweight, normal-weight, overweight and obese women, respectively; high GWG, $>18.0,>16.0,>11.5$ and $>9.0 \mathrm{~kg}$ for underweight, normal-weight, overweight and obese women, respectively; optimal GWG, weight gain between the cut-offs for low and high GWG.

excessive weight gain being $0 \cdot 28$ (95\% CI 0.08, 0.94; $P=0.04)$ and $0.55(95 \% \mathrm{CI} 0.32,0.95 ; P=0.03)$, respectively. Moreover, women with normal BMI and with excessive weight gain during pregnancy $(\mathrm{OR}=1 \cdot 18$; $95 \%$ CI $1.01,1.37 ; P=0.034$ ) were more likely to continue EBF until 6 months postpartum.

\section{Discussion}

We examined the relationship between GWG and EBF for the first 6 months postpartum in 8449 primiparas in rural China. The overall rate of EBF at 6 months was $58.7 \%$, and we found that weight gain below or above guidelines was 
Table 2 OR and $95 \% \mathrm{Cl}$ from two logistic regression models of successful exclusive breast-feeding for the first 6 months among primiparas ( $n$ 8449) from five rural counties in Hebei Province, China, May 2006-April 2009

\begin{tabular}{|c|c|c|c|c|c|c|}
\hline Characteristic & $n$ & Unadjusted OR & $95 \% \mathrm{Cl}$ & Adjusted* OR & $95 \% \mathrm{Cl}$ & $P$ value \\
\hline \multicolumn{7}{|l|}{ GWG† } \\
\hline Optimal & 3410 & 1.00 & Reference & 1.00 & Reference & - \\
\hline Less & 3044 & 0.86 & $0.78,0.95$ & 0.85 & $0.77,0.94$ & 0.002 \\
\hline Excessive & 1995 & 0.95 & $0.85,1.07$ & 1.03 & $0.92,1.16$ & 0.622 \\
\hline \multicolumn{7}{|c|}{ Pre-pregnancy $\mathrm{BMI} \ddagger \times \mathrm{GWG}$} \\
\hline \multicolumn{7}{|c|}{ Normal weight } \\
\hline Optimal & 2493 & 1.00 & Reference & 1.00 & Reference & - \\
\hline Less & 2668 & 0.91 & $0.81,1.01$ & 0.91 & $0.81,1.01$ & 0.086 \\
\hline Excessive & 1059 & & $0.95,1.28$ & $1 \cdot 18$ & $1.01,1.37$ & 0.033 \\
\hline \multicolumn{7}{|c|}{ Underweight } \\
\hline Optimal & 216 & 1.00 & Reference & 1.00 & Reference & - \\
\hline Less & 204 & $1 \cdot 13$ & $0.76,1.66$ & $1 \cdot 12$ & $0.76,1.67$ & 0.563 \\
\hline Excessive & 111 & 1.22 & $0.76,1.95$ & 1.20 & $0.75,1.94$ & 0.451 \\
\hline \multicolumn{7}{|l|}{ Overweight } \\
\hline Optimal & 612 & 1.00 & Reference & 1.00 & Reference & - \\
\hline Less & 158 & 0.49 & $0.34,0.69$ & 0.49 & $0.34,0.70$ & $<0.01$ \\
\hline Excessive & 625 & 0.75 & $0.60,0.94$ & 0.79 & $0.63,0.99$ & 0.048 \\
\hline \multicolumn{7}{|l|}{ Obese } \\
\hline Optimal & 89 & 1.00 & Reference & 1.00 & Reference & - \\
\hline Less & 14 & 0.34 & $0.11,1.09$ & 0.28 & $0.08,0.94$ & 0.040 \\
\hline Excessive & 200 & 0.51 & $0.30,0.86$ & 0.55 & $0.32,0.95$ & 0.030 \\
\hline
\end{tabular}

GWG, gestational weight gain.

${ }^{*}$ Adjusted for confounders, including education, gravidity, caesarean and preterm.

†Low GWG, $<12.5,<11.5,<7.0$ and $<5.0 \mathrm{~kg}$ for underweight, normal-weight, overweight and obese women, respectively; high GWG, $>18.0,>16.0,>11.5$ and $>9.0 \mathrm{~kg}$ for underweight, normal-weight, overweight and obese women, respectively; optimal GWG, weight gain between the cut-offs for low and high GWG.

‡BMI was categorized as underweight $\left(<18.5 \mathrm{~kg} / \mathrm{m}^{2}\right)$, normal weight $\left(18.5-23.9 \mathrm{~kg} / \mathrm{m}^{2}\right)$, overweight $\left(24.0-27.9 \mathrm{~kg} / \mathrm{m}^{2}\right)$ or obese $\left(\geq 28.0 \mathrm{~kg} / \mathrm{m}^{2}\right)$.

associated with EBF behaviour among overweight and obese women.

The interest in EBF for the first 6 months stems from the important benefits it can bring to public health. The rate we obtained for $\mathrm{EBF}$ is substantially higher than the weighted rate $(18.5 \%)$ in rural areas found by a national survey in $2013^{(8)}$. Research suggests that multiparous women are more likely to breast-feed through the first 6 months ${ }^{(31)}$, so the inclusion of primiparas could lead to an underestimation of the actual rate of EBF. The health education and EBF promotion programmes that were in progress in the study area during the randomized controlled trial, which have been shown to promote the continuation of breast-feeding ${ }^{(32)}$, could account for the high rate of EBF found by the present study. Moreover, our participants tended to be well nourished and healthconscious, which might have also had an effect on the rate of EBF. Women without anaemia or with mild anaemia were well nourished and were in healthier physical condition than the average woman in China. The inclusion criterion that dates of menstruation were recorded for at least 2 months before the beginning of the study selected participants who exhibited greater concern about their health.

Previous studies have focused on excessive weight gain, a more interesting and epidemiological topic, but our results on low weight gain are consistent with many of those studies. Winkvist et al. ${ }^{(22)}$ indicated that, among overweight and obese pregnant Norwegian women, the possibility of being unable to sustain full breast-feeding for 6 months was higher when GWG was below or above the recommended levels. In that study, full breast-feeding was used instead of EBF because not all versions of their questionnaire included questions on use of water, waterbased drinks or fruit juice. It can be inferred that the actual $\mathrm{EBF}$ rate would be lower than the full breast-feeding rate $(13.7 \%)$. This difference in outcome variable had little influence on the association between GWG and breastfeeding.

Using hazard analysis, Hilson et al. ${ }^{(20)}$ found that prepregnant obesity and excessive GWG were both significant factors for discontinuing EBF among white women, and they found no interaction between these variables. Obese women with low weight gain were also more likely to discontinue EBF. This supported the negative effect, but the duration for EBF in that study was less than 8 weeks ( 2 months) for most participants, quite a bit shorter than our time horizon of 6 months postpartum. As in another study conducted in Brazil ${ }^{(21)}$, obese pregnant women were more likely to introduce supplementary, non-breast foods. The same was true for overweight and obese women who gained excessive weight. That study observed EBF over the first month of life and found a rate of $\mathrm{EBF}$ of $77.4 \%$. It is to be regretted that no longer-term 
study of EBF has been performed. The three studies cited above examined normal pre-pregnancy BMI with optimal GWG as the reference in regression models. However, in our study, we examined the association for four prepregnancy BMI categories and used optimal GWG for each category as the reference. BMI before pregnancy was taken as a characteristic of each woman and GWG was used as the controllable factor that occurred during pregnancy. Moreover, in clinical practice, doctors use prepregnancy BMI as a guideline for instructing pregnant women on the desirable amount of weight gain they should experience during pregnancy, so we compared women who either failed to reach the recommended weight or exceeded it during pregnancy with those who sustained optimal GWG, within four BMI groups.

A prospective birth cohort study in China examined the influence of maternal pre-pregnancy BMI and GWG on the duration of infant breast-feeding, and no differences in duration of EBF according to pre-pregnancy BMI or GWG were found. The classification criteria for GWG may have accounted for the difference. That cohort study used Chinese-recommended weight gain standards derived from 76854 individuals: underweight women are instructed to gain $15 \cdot 0-22 \cdot 0 \mathrm{~kg}$ during pregnancy; normal-weight women should gain $13.0-21.0 \mathrm{~kg}$; overweight women are told to gain 10.0-18.0 kg; and obese women are given 9.5$17.0 \mathrm{~kg}$ as their target ${ }^{(33)}$. The recommended weight gain for this standard was significantly higher than the Institute of Medicine criteria and evidence that the former one seemed more suitable for Chinese was deficient.

The association between weight gain during gestation and EBF could be explained as follows. First, gaining more weight during pregnancy is associated with increased risk for gestational diabetes mellitus ${ }^{(34)}$, which is considered a risk factor for non-elective caesarean section ${ }^{(35)}$, a practice that adversely affects $\mathrm{EBF}^{(36)}$. Moreover, women with indicators of insulin resistance have a significantly lower rate of milk production, leading to insufficient milk sup$\mathrm{ply}^{(37)}$. Second, poor body image due to excessive weight gain is related to a lack of confidence and comfort with breast-feeding among primiparas ${ }^{(38,39)}$. Finally, few studies have focused on low GWG and EBF, but there is moderate evidence that inadequate weight gain is associated with decreased initiation of breast-feeding ${ }^{(40)}$.

Our study used trained staff who used standardized equipment and methods to measure weight and height during prenatal examinations rather than documenting self-reported weight, thus preventing category misclassification. However, there were also several limitations to the present study. First, as described above, our study population was drawn from a randomized controlled trial whose participants tended to be health-conscious. However, we do not believe that aspect of our study was sufficient to be a confounding factor affecting the associations we found. Second, our study did not take account of potential psychological factors, such as breast-feeding intention or self-efficacy, although prenatal health education was regularly performed. Third, we used weight values that were measured at the first prenatal care visit to calculate pre-pregnancy BMI and GWG. This figure may overestimate pre-pregnancy BMI and underestimate GWG. We reviewed pre-pregnancy weight records where available and found that the mean weight differences between pre-pregnancy and the prenatal visit weights were less than $1 \mathrm{~kg}$. A previous study also used a figure for weight collected at the first prenatal care visit to calculate pre-pregnancy BMI and $\mathrm{GWG}^{(41)}$.

In summary, having a GWG below or above Institute of Medicine recommendations is associated with EBF practices for the first 6 months postpartum among overweight and obese rural primiparas in China. Due to the large sample size, when reading the results, attention should be paid not only to statistical significance, but also to the effect size, that is how far OR differ from 1. Optimal GWG should be maintained during pregnancy, particularly for overweight and obese women. Relevant health educational and nutritional interventions should be conducted to promote and support EBF.

\section{Acknowledgements}

Acknowledgements: The authors thank the hundreds of health-care workers in the Maternal and Child Health stations in the five target counties (Xianghe, Fengrun, Laoting, Mancheng and Yuanshi) in Hebei Province during 2006 through 2009, members of the data safety and monitoring board, and all other staff who participated in this project. Financial support: The study was supported by a cooperative agreement between Peking University Health Science Center and the Centers for Disease Control and Prevention. The funders had no role in the design, analysis or writing of this article. Conflict of interest: None. Authorship: M.T. and L.J. conceived the idea of the manuscript and led the analysis, interpretation and writing of the manuscript. M.T. analysed the data and wrote the paper. Other co-authors contributed to the review of the manuscript. Ethics of human subject participation: This study was conducted according to the guidelines laid down in the Declaration of Helsinki and all procedures involving human subjects were approved by the institutional review boards of the Centers of Disease Control and Prevention, Atlanta, GA, USA and Peking University, Beijing, China. The consent forms were signed by the physician plus a witness after women provided verbal consent during the first prenatal visit.

\section{Supplementary material}

To view supplementary material for this article, please visit https://doi.org/10.1017/S1368980019000387 


\section{References}

1. World Health Organization \& UNICEF (2003) Global Strategy for Infant and Young Child Feeding. http://apps.who. int/iris/bitstream/handle/10665/42590/9241562218.pdf (accessed December 2018).

2. Ip S, Chung M, Raman G et al. (2007) Breastfeeding and maternal and infant health outcomes in developed countries. Evid Rep Technol Assess (Full Rep) issue 153, 1-186.

3. Chowdhury R, Sinha B, Sankar MJ et al. (2015) Breastfeeding and maternal health outcomes: a systematic review and meta-analysis. Acta Paediatr 104, 96-113.

4. Zheng JS, Liu H, Li J et al. (2014) Exclusive breastfeeding is inversely associated with risk of childhood overweight in a large Chinese cohort. J Nutr 144, 1454-1459.

5. Krawczyk A, Lewis MG, Venkatesh BT et al. (2016) Effect of exclusive breastfeeding on rotavirus infection among children. Indian J Pediatr 83, 220-225.

6. Lin H, Sun L, Lin J et al. (2014) Protective effect of exclusive breastfeeding against hand, foot and mouth disease. $B M C$ Infect Dis 14, 645.

7. Victora CG, Bahl R, Barros AJ et al. (2016) Breastfeeding in the 21st century: epidemiology, mechanisms, and lifelong effect. Lancet 387, 475-490.

8. Duan Y, Yang Z, Lai J et al. (2018) Exclusive breastfeeding rate and complementary feeding indicators in China: a national representative survey in 2013. Nutrients 10, E249.

9. Guo S, Fu X, Scherpbier RW et al. (2013) Breastfeeding rates in central and western China in 2010: implications for child and population health. Bull World Health Organ 91, 322-331.

10. Liu P, Qiao L, Xu F et al. (2013) Factors associated with breastfeeding duration: a 30-month cohort study in northwest China. J Hum Lact 29, 253-259.

11. Liu S, Li J, Gong C et al. (2014) Evaluation of the feeding status of infants and young children under 2 years old in rural areas of Hubei province. Zhonghua Yu Fang Yi Xue Za Zhi 48, 705-709.

12. Qu P, Wang T, Liu F et al. (2015) Breast-feeding patterns of ethnic groups in rural western China. Public Health Nutr 18, 3386-3393.

13. Zhang LF, Feng S \& Wen F (2016) Analysis of the status and influencing factors of breastfeeding among infants within six months of ages in Haidian neighbourhood of Haidian district of Beijing. Chin J Child Health Care 24, 1081-1083.

14. Su SG, Qu WJ, Ni B et al. (2017) Status quo and influencing factors of breastfeeding among infants aged 6-month and elder in Dalian municipality. Chin J Public Health 33, 792-795.

15. Boccolini CS, Carvalho ML \& Oliveira MI (2015) Factors associated with exclusive breastfeeding in the first six months of life in Brazil: a systematic review. Rev Saude Publica 49, 91.

16. Chaves RG, Lamounier JA \& Cesar CC (2007) Factors associated with duration of breastfeeding. J Pediatr (Rio J) 83, 241-246.

17. Machado MC, Assis KF, Oliveira Fde C et al. (2014) Determinants of the exclusive breastfeeding abandonment: psychosocial factors. Rev Saude Publica 48, 985-994.

18. Pang WW, Aris IM, Fok D et al. (2016) Determinants of breastfeeding practices and success in a multi-ethnic Asian population. Birth $\mathbf{4 3}, 68-77$.

19. Tang L, Lee AH \& Binns CW (2015) Factors associated with breastfeeding duration: a prospective cohort study in Sichuan Province, China. World J Pediatr 11, 232-238.

20. Hilson JA, Rasmussen KM \& Kjolhede CL (2006) Excessive weight gain during pregnancy is associated with earlier termination of breast-feeding among white women. $J$ Nutr 136, 140-146.

21. Fernandes TA, Werneck GL \& Hasselmann MH (2012) Prepregnancy weight, weight gain during pregnancy, and exclusive breastfeeding in the first month of life in Rio de Janeiro, Brazil. J Hum Lact 28, 55-61.

22. Winkvist A, Brantsaeter AL, Brandhagen M et al. (2015) Maternal prepregnant body mass index and gestational weight gain are associated with initiation and duration of breastfeeding among Norwegian mothers. J Nutr 145, $1263-1270$.

23. Li R, Jewell S \& Grummer-Strawn L (2003) Maternal obesity and breast-feeding practices. Am J Clin Nutr 77, 931-936.

24. Manios Y, Grammatikaki E, Kondaki K et al. (2009) The effect of maternal obesity on initiation and duration of breast-feeding in Greece: the GENESIS study. Public Health Nutr 12, 517-524.

25. Bartok CJ, Schaefer EW, Beiler JS et al. (2012) Role of body mass index and gestational weight gain in breastfeeding outcomes. Breastfeed Med 7, 448-456.

26. Castillo H, Santos IS \& Matijasevich A (2016) Maternal prepregnancy BMI, gestational weight gain and breastfeeding. Eur J Clin Nutr 70, 431-436.

27. Baker JL, Michaelsen KF, Sorensen TIA et al. (2007) High prepregnant body mass index is associated with early termination of full and any breastfeeding in Danish women. Am J Clin Nutr 86, 404-411.

28. Tao XY, Huang K, Yan SQ et al. (2017) Pre-pregnancy BMI, gestational weight gain and breast-feeding: a cohort study in China. Public Health Nutr 20, 1001-1008.

29. Zhou YB, Li HT, Ye RW et al. (2017) Gestational weight gain, cesarean delivery, and cesarean delivery on maternal request: a cohort analysis of Chinese nulliparous women. Ann Epidemiol 27, 96.e3-102.e3.

30. Liu JM, Mei Z, Ye R et al. (2013) Micronutrient supplementation and pregnancy outcomes: double-blind randomized controlled trial in China. JAMA Intern Med $\mathbf{1 7 3}$, 276-282.

31. Hackman NM, Schaefer EW, Beiler JS et al. (2015) Breastfeeding outcome comparison by parity. Breastfeed Med 10, $156-162$.

32. Sun K, Chen M, Yin Y et al. (2017) Why Chinese mothers stop breastfeeding: mothers' self-reported reasons for stopping during the first six months. J Child Health Care 21, 353-363.

33. Yang S, Peng A, Wei S et al. (2015) Pre-pregnancy body mass index, gestational weight gain, and birth weight: a cohort study in China. PLoS One 10, e0130101.

34. Zhong C, Li X, Chen R et al. (2017) Greater early and midpregnancy gestational weight gain are associated with increased risk of gestational diabetes mellitus: a prospective cohort study. Clin Nutr ESPEN 22, 48-53.

35. Gorgal R, Goncalves E, Barros M et al. (2012) Gestational diabetes mellitus: a risk factor for non-elective cesarean section. J Obstet Gynaecol Res 38, 154-159.

36. Zhao J, Zhao Y, Du M et al. (2017) Does caesarean section affect breastfeeding practices in China? A systematic review and meta-analysis. Matern Child Health J 21, 2008-2024.

37. Riddle SW \& Nommsen-Rivers LA (2017) Low milk supply and the pediatrician. Curr Opin Pediatr 29, 249-256.

38. Newby RM \& Davies PS (2016) Antenatal breastfeeding intention, confidence and comfort in obese and non-obese primiparous Australian women: associations with breastfeeding duration. Eur J Clin Nutr 70, 935-940.

39. Hauff LE \& Demerath EW (2012) Body image concerns and reduced breastfeeding duration in primiparous overweight and obese women. Am J Hum Biol 24, 339-349.

40. Institute of Medicine \& National Research Council Committee to Reexamine IOM Pregnancy Weight Guidelines (2009) Weight Gain During Pregnancy: Reexamining the Guidelines. Washington, DC: National Academies Press.

41. Martinez JL, Chapman DJ \& Perez-Escamilla R (2016) Prepregnancy obesity class is a risk factor for failure to exclusively breastfeed at hospital discharge among Latinas. J Hum Lact 32, 258-268. 